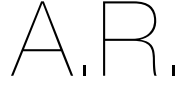

ARTIGO DE REVISÃO

Escola Superior de Tecnologia da Saúde de Lisboa do Instituto Politécnico de Lisboa, Av. D. João Il, Lote 4.69 .01

1900-096 Lisboa, Portuga

2 (H\&TRC) Health \& Technology Research Center, (ESTeSL) Escola Superior de Tecnologia da Saúde de Lisboa, (IPL) Instituto Politécnico de Lisboa,

Av. D. João II, Lote 4.69.01,

1900-096 Lisboa, Portugal

"Endereço para correspondência:

Catarina L

Escola Superior de Tecnologia da Saúde de Lisboa do Instituto Politécnico de Lisboa, Av. D. João II, Lote 4.69.01, 1900-096 Lisboa, Portugal catarinali@hotmail.com

Histórico do artigo:

Recebido a 29 de março de 2021 Aceite a 15 de setembro de 202

\title{
HIGHLIGHTS DA SUPLEMENTAÇÃO DE VI- TAMINA D NOS DOENTES COM COVID-19 - REVISÃO DA EVIDÊNCIA CIENTÍFICA
}

\author{
HIGHLIGHTS OF VITAMIN D SUPPLEMENTATION IN PATIENTS \\ WITH COVID-19 - REVIEW OF SCIENTIFIC EVIDENCE
}

Catarina Li' ${ }^{1 *}$ Inês Gamboa Henriques'; Marisa Cebola²

RESUMO

INTRODUÇÃo: A pandemia da COVID-19 tem levantado discussões acerca dos benefícios da vitamina D na prevenção e no tratamento da doença. Esta vitamina desempenha um papel eficaz no sistema imunológico, podendo contribuir para uma resposta adequada à infeção por SARS-CoV-2.

OBJETIVOS: Rever a evidência científica sobre o efeito da suplementação da vitamina D nos doentes com COVID-19. METODOLOGIA: A pesquisa foi realizada em bases de dados eletrónicas, nomeadamente Pubmed e Web of Science, com publicações no último ano, utilizando as palavras-chave: "COVID-19" or "SARS-CoV-2" or "coronavirus", vitamin D" and "dietary supplements". Após aplicar os critérios de inclusão e exclusão, obtiveram-se 9 artigos.

RESULTADOS: A suplementação da vitamina D nos doentes com COVID-19 reduziu o tempo de internamento hospitalar, a necessidade de admissão nos cuidados intensivos e de ventilação mecânica invasiva, acelerando o processo de recuperação. Estudos relatam os possíveis benefícios da suplementação como medida profilática de forma a proteger contra futuras infeções víricas.

CONCLUSÕES: A evidência científica existente demonstra resultados promissores do papel da vitamina D nos doentes com COVID-19 na redução da gravidade da doença e na melhoria do prognóstico. São necessários mais estudos na população humana para suportar esta hipótese e comprovar a eficácia.

\section{PALAVRAS-CHAVE}

Coronavirus, COVID-19, Dietary supplements, Vitamina D, SARS-CoV-2

\section{ABSTRACT}

INTRODUCTION: The COVID-19 pandemic has raised discussions about the benefits of vitamin D in preventing and treating the disease. This vitamin plays an effective role in the immune system and can contribute to an adequate response to SARS-CoV-2 infection. The purpose of this review is to systematize the scientific evidence on the effect of vitamin D in the patients with COVID-19. OBJECTIVES: Review the scientific evidence on the effect of vitamin D in the patients with COVID-19.

METHODOLOGY: The search was carried out in electronic databases, namely Pubmed, and Web of Science, with publications in the last year, using the keywords: "COVID-19" or "SARS-CoV-2" or "coronavirus", vitamin D" and "dietary supplements". After applying the inclusion and exclusion criteria, 9 articles were obtained.

RESULTS: Supplementation of vitamin D in patients with COVID-19 reduced the length of hospital stay, the need for admission to intensive care and invasive mechanical ventilation, accelerating the recovery process. Studies report the possible benefits of supplementation as a prophylactic measure in order to protect against future viral infections.

CONCLUSIONS: Scientific evidence demonstrates promising results from the role of vitamin D in patients with COVID-19 in reducing the severity of the disease and improving the prognosis. Further studies in the human population are needed to support this hypothesis and prove its effectiveness.

KEYWORDS

Coronavirus, COVID-19, Dietary supplements, Vitamin D, SARS-CoV-2

\section{INTRODUÇÃO}

As infeções agudas do trato respiratório constituem uma das principais causas de morbidade e mortalidade em todo o mundo, conforme comprovado por ambas as pandemias de influenza sazonal e o recente surto da Coronavirus disease 2019 (COVID-19), causada pela infeção por Severe acute respiratory syndrome coronavírus 2 (SARS-CoV-2) (1). É indubitável que o maior desafio para deter esta pandemia é a ausência de evidências demonstrando intervenções farmacológicas eficazes para prevenir a COVID-19. Contudo, sabe-se que numa infeção vírica, é fundamental otimizar a função do sistema imunológico $(2,3)$. Um estado nutricional debilitado conduz à carência de vários micronutrientes, tais como vitaminas A, D, C, E, B6, B12, folato, cobre, ferro, zinco e selénio, que são fulcrais para o normal funcionamento 
do sistema imunológico (2-5). Assim sendo, a correção de todas as carências nutricionais e não apenas de um nutriente é essencial para uma resposta adequada à infeção pelo SARS-CoV-2 $(3,4)$.

A pandemia da COVID-19 tem levantado discussões acerca dos benefícios da vitamina $D$ na prevenção e no tratamento da doença. $A$ razão mais plausível consiste no facto de níveis suficientes de vitamina D no sangue desempenharem um papel eficaz no funcionamento do sistema imunológico, sustentando uma resposta celular satisfatória na proteção contra a gravidade das infeções causadas por microorganismos (6). Deste modo, a vitamina $D$ exerce um papel imunomodulador, aumentando a imunidade inata pela secreção de peptídeos antivirais (7).

Neste sentido, a deficiência de vitamina $D(25(\mathrm{OH}) \mathrm{D}<20 \mathrm{nmolL})$ foi associada a uma maior gravidade da COVID-19 e a piores outcomes, levantando várias discussões sobre os potenciais benefícios da suplementação desta vitamina no tratamento desta patologia (6).

O presente artigo de revisão tem como objetivo rever a evidência científica sobre os efeitos da suplementação da vitamina $D$ em doentes com COVID-19, de modo a analisar literatura existente acerca deste recente tema.

\section{METODOLOGIA}

A pesquisa do artigo foi realizada em base de dados eletrónicas, de busca e recuperação de artigos na área das ciências da vida, nomeadamente Pubmed e Web of Science, entre janeiro e fevereiro de 2021. Foram selecionadas palavras-chave como "COVID-19" or "SARSCoV-2" or "coronavirus", and "vitamin D" and "dietary supplements". Os critérios de inclusão focaram-se em artigos publicados no último ano (2020-2021 inclusive), estudos do tipo ensaio clínico, ensaios clínicos randomizados, estudos coorte e estudos observacionais (seguindo os critérios da check-list PRISMA (8)), artigos relacionados com o tema em causa e publicações na língua portuguesa e inglesa. No que diz respeito aos critérios de exclusão, foram excluídos revisões sistemáticas e meta-análises, estudos realizados em animais, publicações anteriores ao ano de 2020 e que não estão relacionados com a temática em estudo (Figura 1).

\section{RESULTADOS}

Na primeira pesquisa foram selecionados 337 artigos. Após retirar os duplicados incluíram-se 334 artigos para a triagem. Após a aplicação dos vários filtros (anos de publicação, tipo de estudo, língua e estudos realizados em humanos), obtiveram-se 18 artigos. Após a leitura do título e abstract, obtiveram-se 12 e após a leitura integral selecionaram-se no final 9 artigos que se encontram apresentados na Tabela 1.

\section{ANÁLISE CRÍTICA}

A evidência científica atual sugere a importância da suplementação da Vitamina $D$ na redução da gravidade da doença e do tempo de internamento hospitalar de doentes COVID-19 com carência desta vitamina, mais concretamente nos doentes críticos internados em cuidados intensivos.

A maioria dos estudos analisados refere de forma clara a suplementação efetuada pelos doentes (dose, período de tempo e frequência) mas o facto de o tipo de estudo (ensaio clínico, ensaios clínicos randomizados, estudos coorte e estudos observacionais) e as amostras não serem sobreponíveis (idosos institucionalizados, idosos em internamento hospitalar e na comunidade) limita as conclusões e a extrapolação para a população em geral.

\section{Figura 1}

Fluxograma PRISMA

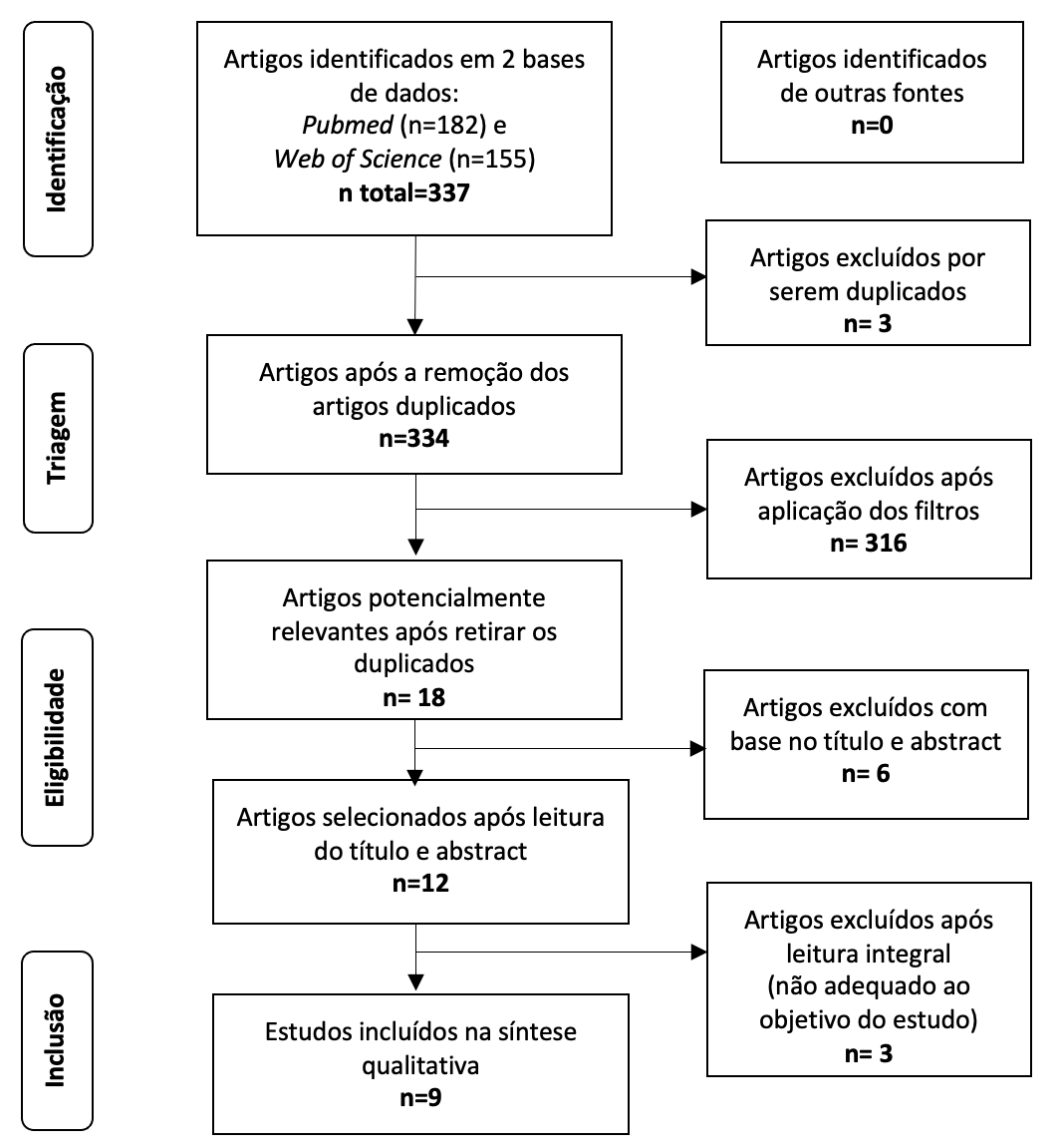


Tabela 1

Resumo dos artigos selecionados

\begin{tabular}{|c|c|c|c|c|c|}
\hline AUTORES & TIPOLOGIA DE ESTUDO & AMOSTRA & OBJETIVO & MÉTOdOS & RESULTADOS \\
\hline $\begin{array}{l}\text { Castillo ME, et al. } \\
(2020)(9)\end{array}$ & ECR- estudo piloto & $\begin{array}{l}76 \text { utentes hospitalizados } \\
\text { com COVID-19 }\end{array}$ & $\begin{array}{l}\text { Avaliar o efeito do tratamento } \\
\text { com calcifediol na unidade } \\
\text { de cuidados intensivos a } \\
\text { mortalidade entre pacientes } \\
\text { hospitalizados com } \\
\text { COVID-19. }\end{array}$ & $\begin{array}{l}\text { Grupo de intervenção: } \\
0,532 \mathrm{mg} \text { de calcifediol oral } \\
\text { na admissão; } 0,266 \mathrm{mg} \text { de } \\
\text { calcifediol oral nos dias } 3 \text { e } 7 \text {, } \\
\text { e a seguir, semanalmente até } \\
\text { à alta ou admissão na UCl. } \\
\text { Grupo sem receber } \\
\text { tratamento com calcifediol. }\end{array}$ & $\begin{array}{l}\text { Nos pacientes que } \\
\text { necessitaram de } \\
\text { hospitalização, a } \\
\text { administração de uma alta } \\
\text { dose de calcifediol ou } 25(\mathrm{OH}) \\
\text { D reduz significativamente a } \\
\text { necessidade de tratamento } \\
\text { na UCl. }\end{array}$ \\
\hline $\begin{array}{l}\text { Ling F.S. et al. } \\
(2020)(10)\end{array}$ & Observacional & $\begin{array}{l}986 \text { participantes } \\
\text { com COVID-19 }\end{array}$ & $\begin{array}{l}\text { Determinar se a mortalidade } \\
\text { associada à COVID-19 é } \\
\text { afetada por níveis séricos de } \\
25(\mathrm{OH}) \mathrm{D} \text {, status de vitamina D } \\
\text { ou terapia com colecalciferol. }\end{array}$ & $\begin{array}{l}\text { Doentes com insuficiência } \\
\text { de vitamina } D(25(\mathrm{OH}) \mathrm{D} \text { entre } \\
25-50 \mathrm{nmol} / \mathrm{L}) \text { receberam } \\
\text { terapia de reforço com } \\
\text { colecalciferol ( } \geq 280.000 \mathrm{UI}) \\
\text { num período de } 7 \text { semanas. }\end{array}$ & $\begin{array}{l}\text { A terapia de reforço } \\
\text { com colecalciferol, } \\
\text { independentemente dos níveis } \\
\text { basais de } 25(\mathrm{OH}) \mathrm{D} \text {, parece } \\
\text { estar associada a um risco } \\
\text { reduzido de mortalidade nos } \\
\text { pacientes internados com } \\
\text { COVID-19. }\end{array}$ \\
\hline $\begin{array}{l}\text { Radujkovic A, et al. } \\
(2020)(11)\end{array}$ & $\begin{array}{l}\text { Prospetivo sem } \\
\text { intervenção }\end{array}$ & $\begin{array}{l}185 \text { doentes } \\
\text { com COVID-19 }\end{array}$ & $\begin{array}{l}\text { Explorar as possíveis } \\
\text { associações entre o nível de } \\
\text { vitamina } D \text { e a severidade e } \\
\text { sobrevivência dos doentes } \\
\text { com COVID-19. }\end{array}$ & $\begin{array}{l}\text { Nível de vitamina } D \\
\text { Carência: }<12 \mathrm{ng} / \mathrm{mL}\end{array}$ & $\begin{array}{l}\text { Carência de vitamina } D \\
\text { associada a um elevado risco } \\
\text { de VMI e mortalidade. }\end{array}$ \\
\hline $\begin{array}{l}\text { Jain A, et al. } \\
(2020)(12)\end{array}$ & $\begin{array}{l}\text { Prospetivo } \\
\text { observacional }\end{array}$ & $\begin{array}{c}154 \text { doentes } \\
\text { com CoVID-19 (30-60 anos) }\end{array}$ & $\begin{array}{l}\text { Analisar o nível de vitamina D } \\
\text { em doentes com COVID-19 e } \\
\text { o impacto na severidade da } \\
\text { doença. }\end{array}$ & $\begin{array}{l}\text { Medição da concentração } \\
\text { de } 25(\mathrm{OH}) \mathrm{D}, \mathrm{IL}-6, \mathrm{~T} \text {, TNF-a e } \\
\text { ferritina. } \\
\text { Grupo A - assintomáticos } \\
\text { Grupo B - severos com } \\
\text { admissão na UCl }\end{array}$ & $\begin{array}{l}\text { Os doentes com COVID-19 } \\
\text { mais severo, admitidos na } \\
\text { UCI, revelaram uma carência } \\
\text { superior de vitamina D. } \\
\text { Marcadores inflamatórios } \\
\text { e taxa de mortalidade mais } \\
\text { elevados nesses doentes. }\end{array}$ \\
\hline $\begin{array}{l}\text { Tan WC, et al. } \\
(2020)(13)\end{array}$ & Coorte Observacional & $\begin{array}{l}43 \text { doentes hospitalizados } \\
\text { com COVID-19 ( } \geq 50 \text { anos) }\end{array}$ & $\begin{array}{l}\text { Determinar as consequências } \\
\text { clínicas da suplementação } \\
\text { oral com vitamina D, } \\
\text { magnésio e vitamina B12 nos } \\
\text { doentes mais velhos com } \\
\text { COVID-19 e a sua relação } \\
\text { com a necessidade de } \\
\text { cuidados intensivos. }\end{array}$ & $\begin{array}{l}\text { Administração oral de } \\
1000 U \mathrm{Ul} / \text { dia de vitamina D3, } \\
150 \mathrm{mg} / \text { dia de magnésio e } \\
500 \mu \mathrm{g} / \text { dia de vitamina B12 } \\
\text { durante } \geq 14 \text { dias, no caso } \\
\text { de não necessitarem de } \\
\text { oxigenoterapia. }\end{array}$ & $\begin{array}{l}\text { Os doentes que receberam } \\
\text { a suplementação revelaram } \\
\text { ter uma menor probabilidade } \\
\text { de necessitarem de } \\
\text { oxigenoterapia em } \\
\text { comparação com o controlo. }\end{array}$ \\
\hline $\begin{array}{l}\text { Annweiler C, et al. } \\
(2020)(14)\end{array}$ & Quase experimental & $\begin{array}{l}66 \text { idosos com COVID-19 } \\
\text { residentes num lar }\end{array}$ & $\begin{array}{l}\text { Determinar se a } \\
\text { suplementação de vitamina } \\
\text { D3 por bolus tomada durante } \\
\text { ou imediatamente antes } \\
\text { da COVID-19 é eficaz em } \\
\text { melhorar a sobrevivência } \\
\text { dos idosos frágeis residentes } \\
\text { em lares }\end{array}$ & $\begin{array}{l}\text { Grupo de intervenção: } \\
\text { suplementação de } 80.000 U \text { I } \\
\text { vitamina D3 em bolus na } \\
\text { semana seguinte à suspeita, } \\
\text { ou após o diagnóstico de } \\
\text { COVID-19 ou durante o mês } \\
\text { anterior; } \\
\text { Grupo de comparação: sem } \\
\text { suplementação. }\end{array}$ & $\begin{array}{l}\text { A suplementação de vitamina } \\
\text { D3 por bolus tomada durante } \\
\text { ou logo antes em idosos } \\
\text { frágeis com COVID-19 foi } \\
\text { associada a menor severidade } \\
\text { da doença e maior taxa de } \\
\text { sobrevivência. }\end{array}$ \\
\hline $\begin{array}{l}\text { Annweiler C, et al. } \\
(2020)(14)\end{array}$ & Quase experimental & $\begin{array}{l}77 \text { idosos com COVID-19 } \\
\text { hospitalizados }\end{array}$ & $\begin{array}{l}\text { Determinar se a } \\
\text { suplementação em bolus } \\
\text { de vitamina } D \text {, tomada } \\
\text { regularmente durante o ano } \\
\text { anterior ou após o diagnóstico } \\
\text { de COVID-19, é eficaz na } \\
\text { melhoria da sobrevivência dos } \\
\text { idosos frágeis com COVID-19 } \\
\text { hospitalizados. }\end{array}$ & $\begin{array}{l}\text { Grupo 1: vitamina D3 por } \\
\text { bolus no ano anterior ( } 50000 \\
\text { UI por mês, ou } 80.000 \text { UI ou } \\
10.0000 \text { UI a cada 2-3 meses). } \\
\text { Grupo 2: vitamina D3 após o } \\
\text { diagnóstico ( } 80.000 \text { UI). } \\
\text { Grupo 3: controlo; não } \\
\text { receberam suplementação. }\end{array}$ & $\begin{array}{l}\text { A suplementação regular } \\
\text { de vitamina D3 por bolus } \\
\text { foi associada a um melhor } \\
\text { prognóstico de COVID-19 e } \\
\text { a uma taxa de sobrevivência } \\
\text { superior. }\end{array}$ \\
\hline $\begin{array}{l}\text { Baktash V, et al. } \\
(2020)(16)\end{array}$ & Coorte Prospetivo & $\begin{array}{c}105 \text { idosos } \\
\text { com COVID-19 ( } \geq 65 \text { anos) }\end{array}$ & $\begin{array}{l}\text { Determinar se os idosos com } \\
\text { maior probabilidade de terem } \\
\text { deficiência de vitamina } D \\
\text { apresentam piores resultados } \\
\text { com COVID-19 }\end{array}$ & $\begin{array}{l}\text { Grupo positivo para COVID-19 } \\
\text { ( } n=70) \text { : } \\
\text { - Défice de vitamina D } \\
\text { ( } \leq 30 \mathrm{nmol} / \mathrm{L})(\mathrm{n}=39) \\
\text { - Sem défice de vitamina D } \\
\geq 30 \mathrm{nmol})(\mathrm{n}=31) \\
\text { Grupo negativo para } \\
\text { COVID-19 }(n=35)\end{array}$ & $\begin{array}{l}\text { Os idosos com deficiência } \\
\text { de vitamina } \mathrm{D} \text { e infetados } \\
\text { com COVID-19 podem } \\
\text { apresentar piores resultados } \\
\text { de morbidade. }\end{array}$ \\
\hline $\begin{array}{l}\text { Meltzer DO, et al. } \\
(2020)(17)\end{array}$ & Coorte retrospetivo & $\begin{array}{c}489 \text { utentes } \\
\text { (média de idades } 49,2 \text { anos) }\end{array}$ & $\begin{array}{l}\text { Examinar se o último nível } \\
\text { doseamento de vitamina } D \\
\text { antes da realização do teste } \\
\text { de COVID-19 está associado } \\
\text { aos seus resultados. }\end{array}$ & $\begin{array}{l}\text { Teste COVID-19 } \\
\text { Medição dos níveis de } \\
\text { vitamina D: } \\
\text { Carência: }<20 \mathrm{ng} / \mathrm{mL}\end{array}$ & $\begin{array}{l}\text { Utentes com níveis baixos } \\
\text { de vitamina } D \text { no momento } \\
\text { da realização do teste } \\
\text { de COVID-19, têm maior } \\
\text { risco de testar positivo do } \\
\text { que indivíduos com níveis } \\
\text { adequados. }\end{array}$ \\
\hline
\end{tabular}

ECR: Ensaio clínico randomizado

IL-6: Interleucina-6

TNF-a: fator de necrose tumoral-a

NR: Não respostado

VMI: Ventilação mecânica invasiva

UCI: Unidade de Cuidados Intensivos

25(OH)D: 25-hidroxivitamina D 
Castillo ME, et al. (9) avaliaram o efeito do tratamento com calcifediol (ou 25(OH)D) na unidade de cuidados intensivos ( $\mathrm{UCl}$ ) e a mortalidade entre doentes hospitalizados com COVID-19. Os doentes tomaram calcifediol oral na admissão (0,532mg) e nos dias 3 e 7 (0,266mg), e a seguir, semanalmente até à alta ou admissão na UCI. Entre os 26 pacientes não tratados, 13 (ou 50\%) necessitaram de ser internados na $\mathrm{UCl}$, enquanto dos 50 doentes tratados, apenas 1 necessitou de internamento na $\mathrm{UCl}$. Ainda dos doentes tratados com calcifediol, nenhum morreu e todos receberam alta hospitalar (sem complicações), enquanto dos 13 pacientes internados na UCI, 2 morreram e 11 receberam alta. Estes resultados indicaram que o calcifediol parece ser capaz de reduzir a gravidade da doença, e deste modo diminuir significativamente a necessidade de os doentes serem internados na UCl. Estes dados vão ao encontro com o estudo realizado por Ling F.S. et al., (10) em que foi analisada a possível associação entre a mortalidade por COVID-19 e os níveis séricos de 25(OH)D, status de vitamina D ou terapia com colecalciferol. Foram incluídos 986 participantes com COVID-19 dos quais 151 (16\%) receberam terapia de reforço com colecalciferol, uma vez que estavam com insuficiência de vitamina $D(25(\mathrm{OH}) \mathrm{D}$ entre $25-50 \mathrm{nmol} / \mathrm{L})$, recebendo $\geq 280.000 \mathrm{Ul}$ de colecalciferol durante 7 semanas. Apesar de já ser expectável, fatores como a idade avançada, doenças isquémicas e creatinina basal elevada $(<83 \mu \mathrm{mol} / \mathrm{L})$ foram associados a um risco superior de morte por COVID-19, dado que estes doentes apresentam um pior estado clínico e menores reservas fisiológicas. Os autores concluíram que a terapia de reforço com colecalciferol, independentemente dos níveis basais de $25(\mathrm{OH}) \mathrm{D}$, parece estar associada a um risco reduzido de mortalidade nos pacientes internados com COVID-19.

Radujkovic A, et al. (11) demonstraram uma associação entre a carência de vitamina $D$ e a gravidade por COVID-19. Utentes com carência de vitamina $D$ tiveram uma taxa de hospitalização superior e necessitaram de mais oxigenoterapia (intensiva) e ventilação mecânica invasiva (VMI). Quando ajustada à idade, sexo e comorbilidades, a carência de vitamina D foi associada a um risco 6 vezes maior da doença severa e um risco 15 vezes maior de morte. Dentro dos 185 participantes do estudo, 22\% e $64 \%$ apresentaram níveis de vitamina D abaixo de 12 e $20 \mathrm{ng} / \mathrm{ml}$, respetivamente. Resultados concordantes foram apresentados por Jain A, et al. (12). Dos 154 doentes com COVID-19 analisados, a carência de vitamina $\mathrm{D}(25(\mathrm{OH}) \mathrm{D}<20 \mathrm{ng} / \mathrm{mL})$ é muito mais prevalente em utentes com COVID-19 grave (grupo B-96,8\%) do que os assintomáticos (grupo A- 32,9\%), que requerem admissão na $\cup \mathrm{Cl}$ e, portanto, têm maiores taxas de mortalidade. Para além disso, esses doentes demonstraram ter valores mais altos dos marcadores inflamatórios (por exemplo níveis de IL-6 e TNF alfa), apresentando uma resposta inflamatória mais intensa. Através destes resultados os autores recomendam a administração de suplementos com vitamina D à população com COVID-19.

No que diz respeito à suplementação, Tan WC, et al. (13) foram comparar os resultados clínicos dos pacientes mais velhos que receberam suplementação oral com 1000 IU/dia de vitamina D, $150 \mathrm{mg} /$ dia de magnésio e 500 $\mu \mathrm{g} /$ dia de vitamina B12 com os que não receberam o suporte referido, e a sua relação com a necessidade de cuidados intensivos. Os investigadores relataram que os doentes que receberam a suplementação tiveram uma menor probabilidade (25\%) de necessitarem de oxigenoterapia em comparação com o controlo (58\%). Para beneficiarem dos efeitos preventivos, os utentes podem necessitar de iniciar a suplementação mais precocemente, na fase infeciosa. A facilidade de administração do suplemento deve permitir este início precoce, ou seja, nos cuidados primários quando há o início dos sintomas ou como profilaxia entre contactos de alto risco durante surtos em grupos identificados. Neste sentido, Annweiler C, et al. (14) investigaram a possível eficácia da suplementação de 80.000UI vitamina D3 por bolus, durante ou imediatamente antes da COVID-19, na melhoria da sobrevivência dos idosos frágeis residentes em lares. Os autores apresentaram que, independentemente de todos os fatores de confusão, a suplementação de vitamina D3 por bolus foi associada a uma menor severidade e maior taxa de sobrevivência nestes doentes, e nenhum outro tratamento mostrou efeito protetor. A severidade da hipovitaminose $D$ parece estar relacionada com o prognóstico de COVID-19, uma vez que, esses doentes tiveram um risco superior de doença severa, de recorrerem e permanecerem mais tempo no internamento. Do mesmo modo, Annweiler G, et al. (15) foram determinar se a suplementação em bolus de vitamina $D$, tomada regularmente durante o ano anterior ou após o diagnóstico de COVID-19, era eficaz na melhoria da sobrevivência dos utentes idosos frágeis com COVID-19 hospitalizados. As conclusões não diferem dos resultados do estudo anterior, os investigadores reportaram que a suplementação regular de vitamina D3 por bolus foi associada a um melhor prognóstico de COVID-19 e a uma taxa de sobrevivência superior. Estes resultados poderão sugerir a possível recomendação da suplementação de vitamina D em todos os idosos, de modo a diminuir a mortalidade.

No que toca aos momentos de testagem para a COVID-19 e a sua relação com os níveis de vitamina D, Baktash V, et al. (14) verificaram que doentes com teste positivo para a COVID-19 possuíam níveis séricos médios inferiores de 25(OH)D (27 nmol/L) quando comparados aos doentes com teste negativo (52 nmol/L), predizendo que $\mathrm{O}$ status de vitamina $D$ pode ser um prognóstico útil. Nos doentes com carência de vitamina $D$ constatou-se uma maior incidência de ventilação mecânica, bem como de maior tempo de internamento (30,77\% vs. 9,68\%). Concordante com estes resultados, o estudo realizado por Meltzer DO, et al. (15) sugere que indivíduos com níveis serológicos baixos de vitamina $\mathrm{D}$, no momento da realização do teste de COVID-19, têm maior risco de testar positivo do que indivíduos com níveis adequados (21,6\% vs. 12,2\%). Nos indivíduos com carência de vitamina $\mathrm{D}$ em que foi aumentado o tratamento, não apresentaram risco superior para a COVID-19 face aos que tinham níveis adequados, indicando um efeito protetor da suplementação de vitamina $\mathrm{D}$. $\mathrm{O}$ baixo custo e a segurança da suplementação, pelo menos em doses até 4.000 Ul por dia, apoiam os argumentos para a suplementação a nível populacional, talvez para grupos de alto risco de carência de vitamina D e/ou COVID-19.

De uma maneira geral, a suplementação com vitamina $\mathrm{D}$ nos doentes com COVID-19 parece ter um efeito promissor. No entanto, dos artigos analisados, alguns estudos apresentaram amostras pequenas, e por isso, não representativas da população, reduzido tempo de follow-up e potenciais fatores de confusão. Para além disso, outros estudos demonstraram suplementação com valores díspares. São necessários mais estudos em humanos, especificamente ensaios clínicos randomizados, com uma amostra representativa e potenciais fatores de confusão e vieses limitados, de forma a aumentar a robustez e a qualidade de futuros estudos.

\section{CONCLUSÕES}

A presente revisão aponta, por um lado, que níveis séricos baixos de vitamina $\mathrm{D}$ relacionam-se com maior risco de testar positivo para a COVID-19 e maior possibilidade de presença de morbilidade, nomeadamente maior necessidade de oxigenoterapia e ventilação mecânica invasiva e, por outro lado que a suplementação de vitamina D nos doentes com COVID-19 parece ter um potencial efeito na redução da gravidade da doença e do tempo de internamento hospitalar, mais concretamente em doentes críticos internados nos cuidados intensivos. $\mathrm{O}$ crescente interesse da comunidade científica relativamente à 
possível relação entre os níveis séricos de vitamina $\mathrm{D}$ com a gravidade e prognóstico da infeção por COVID-19 e também com a imunidade deverá ser consolidada com evidência científica mais robusta para que se possa estabelecer a importância da terapêutica nutricional no tratamento/recuperação dos doentes e na definição de políticas nutricionais que previnam/diminuam a gravidade da doença. A suplementação regular de vitamina $D$ poderá ter um papel importante como medida profilática prevenindo futuras infeções víricas.

\section{REFERÊNCIAS BIBLIOGRÁFICAS}

1. Calder PC, Carr AC, Gombart AF, Eggersdorfer M. Optimal Nutritional Status for a Well-Functioning Immune System Is an Important Factor to Protect against Viral Infections Philip. Nutrients. 2020;12(8):1-3.

2. Iddir M, Brito A, Dingeo G, Del Campo SSF, Samouda H, La Frano MR, et al. Strengthening the immune system and reducing inflammation and oxidative stress through diet and nutrition: Considerations during the COVID-19 crisis. Nutrients. 2020;12(6):1-39.

3. Gasmi A, Tippairote T, Mujawdiya PK, Peana M. Micronutrients as immunomodulatory tools for COVID-19 management. Clin Nutr. 2020;220(January).

4. Gombart AF, Pierre A, Maggini S. A review of micronutrients and the immune system-working in harmony to reduce the risk of infection. Nutrients. 2020;12(1).

5. Jayawardena R, Sooriyaarachchi $P$, Chourdakis M, Jeewandara C, Ranasinghe $P$. Enhancing immunity in viral infections, with special emphasis on COVID-19: A review Ranil. Diabetes Metab Syndr Clin Res Rev. 2020;14(January):367-82.

6. Pereira M, Dantas Damascena A, Galvão Azevedo LM, de Almeida Oliveira T, da Mota Santana J. Vitamin D deficiency aggravates COVID-19: systematic review and meta-analysis. Crit Rev Food Sci Nutr [Internet]. 2020;0(0):1-9. Available from: https:// doi.org/10.1080/10408398.2020.1841090.

7. Ali N. Role of vitamin D in preventing of COVID-19 infection, progression and severity. J Infect Public Health. 2020;(13):1373-80.

8. Liberati A, Altman DG, Tetzlaff J, Mulrow C, Gøtzsche PC, loannidis JPA, et al. The PRISMA statement for reporting systematic reviews and meta-analyses of studies that evaluate health care interventions: Explanation and elaboration. PLoS Med. 2009;6(7). 9. Castillo, Marta Entrenas; Costa, Luis Manuel Entrenas Costa; Barrios, José Manuel Vaquero; Díaz JFA et al. "Effect of calcifediol treatment and best available therapy versus best available therapy on intensive care unit admission and mortality among patients hospitalized for COVID-19: A pilot randomized clinical study." J Steroid Biochem Mol Biol. 2020;203(105751):1-7.

10. Ling SF, Broad E, Murphy R, Pappachan JM, Pardesi-Newton S, Kong MF, et al. High-dose cholecalciferol booster therapy is associated with a reduced risk of mortality in patients with covid-19: A cross-sectional multi-centre observational study. Nutrients. 2020;12(12):1-16.

11. Radujkovic A, Hippchen T, Tiwari-Heckler S, Dreher S, Boxberger M, Merle U. Vitamin D deficiency and outcome of COVID-19 patients. Nutrients. 2020;12(9):1-13. 12. Jain A, Chaurasia R, Sengar NS, Singh M, Mahor S, Narain S. Analysis of vitamin D level among asymptomatic and critically ill COVID-19 patients and its correlation with inflammatory markers. Sci Rep [Internet]. 2020;10(1):1-8. Available from: https://doi. org/10.1038/s41598-020-77093-z.

13. Tan Wen, Chuen; Ho Pock, Liam; Kalimuddin, Shirin; Cherng Pei Zhi, Benjamin; Teh Ean, Yii; Thein Yee S et al. Cohort study to evaluate the effect of vitamin D, magnesium, and vitamin B12 in combination on progression to severe outcomes in older patients with coronavirus (COVID-19). Nutrition. 2020;79-80(111017):1-3.

14. Annweiler, Cédric; Hanotte B et al. Vitamin D and survival in COVID-19 patients : A quasi-experimental study. J Steroid Biochem Mol Biol. 2020;204(105771):1-7. 15. Annweiler G, Corvaisier M, Gautier J, Dubée V, Legrand E, Sacco G, et al. Vitamin d supplementation associated to better survival in hospitalized frail elderly covid-19 patients: The geria-covid quasi-experimental study. Nutrients. 2020;12(11):1-12.

16. Baktash V, Hosack T, Patel N, Shah S, Kandiah P, Van Den Abbeele K, et al. Vitamin D status and outcomes for hospitalised older patients with COVID-19. Postgrad Med J. 2020;2:1-6.
17. Meltzer DO, Best TJ, Zhang H, Vokes T, Arora V, Solway J. Association of Vitamin D Status and Other Clinical Characteristics With COVID-19 Test Results. JAMA Netw open. 2020;3(9):e2019722. 